\title{
A Dynamic Emergency Evacuation Network Optimization Problem with Crossing Elimination Strategy
}

\author{
Wang Ze ${ }^{1, a}$, Ma Shiyong ${ }^{2, b}$, An Shi ${ }^{1, c}$ \\ ${ }^{1}$ School of Transportation Science and Engineering, Harbin Institute of Technology, Harbin, 150090, \\ China \\ ${ }^{2}$ School of Management, Harbin Institute of Technology, Heilongjiang institute of science \\ and technology, Harbin, 150027, China \\ a sunshineym@163.com, ${ }^{b}$ mashiyong1009@126.com, canshi@hit.edu.cn
}

\section{Keywords: Evacuation Planning; Dynamic Traffic Assignment; Cell Transmission Model; Crossing} Elimination

\begin{abstract}
This paper presents a dynamic evacuation network optimization problem that incorporates crossing elimination strategy. Bottleneck problem at intersections during evacuation process is analyzed for the effective evacuation planning. The developed model is formulated as a system-optimum traffic assignment based on cell transmission model (CTM), which can effectively investigate the characteristics of randomness and dynamics of evacuation flow's spatial and temporal distribution. The numerical results show that the model can provide crucial theoretical support for evacuation decision-making for traffic managers.
\end{abstract}

\section{Introduction}

Evacuation has been widely used as an effective emergency response and mitigation strategy to protect people against natural and man-made emergency events, such as hurricanes, wildfires, floods, chemical spills, or terrorist attacks. The overall efficiency of the evacuation plan is a key factor for evacuation success in a large-scale urban region. It is widely known that traffic jams may easily be formed in the intersections of the road network, and intersection capacity may restrict the accessibility of evacuation flow especially when the evacuation demand soars up in the short time. As a matter of fact, traffic signal can separate the conflict flow from different directions by different timings in order to ensure traffic safety and improve operation efficiency. However, under the extreme condition, the signal facilities of the intersection may be destroyed due to the failure of electric power, damage of traffic sensors and communication interruption, and the signal lights cannot be functional described by Ardekani and Hobeika ${ }^{[1]}$. The evacuation may be a new disaster unless the disruptive intersections are not managed well, so the intersection crossing elimination is paying more and more attention in evacuation management in recent years. The primary research was carried out by Cova and Johnson ${ }^{[2]}$ and a lane-based routing plan was generated that traded total vehicle travel-distance against merging, while preventing traffic crossing-conflicts at intersections.

There are some important considerations of implementing the intersection crossing elimination strategy in evacuation planning. First, the abnormally high evacuation demand may easily cause a wide range of traffic congestion and severe delays when the key intersection suffers from little disturbance. Second, it is extremely difficult to make a reasonable signal control plan to maximize the global evacuation efficiency because of the difficulty of an accurate traffic volume prediction, after all the traffic volume may stuck into a unstable fluctuation. Third, drivers under the emergency often become so impatient and panic that they may violate the traffic regulations and drive illegally through a red light timing. This behavior will greatly reduce the evacuation efficiency and traffic safety of the intersection.

The theoretical basis of the evacuation strategy is to grasp the evacuees' behavior and characteristics of evacuation traffic flow. At present, domestic and foreign scholars on disaster management caused by large-scale regional evacuation modeling approach adopted is broadly divided 
into: network flow theory, traffic assignment theory and computer simulation method based on a simple path selection rules ${ }^{[3-6]}$. The paper discusses the intersection crossing elimination strategy in a traffic assignment frame based on modified cell transmission model (CTM), which can effectively investigate the characteristics of randomness and dynamics of evacuation flow's spatial and temporal distribution. The cell transmission model is proposed by Daganzo ${ }^{[7]}$, which is firstly used to simulate a highway traffic flow, and applied into the transport network. Compared with node-arc network, cell network description can better express the transmission and evolution mechanism of evacuation traffic flow, including potential queue forming and dissipation. We conduct our study in the following sequence. Crossing elimination methods are first analyzed and then cell network with is described. Secondly we formulate a cell-based evacuation network optimization model with the crossing elimination strategy. Finally, our case and its results are showed in the last section.

\section{Evacuation optimization model with crossing elimination strategy}

Evacuation optimization model based on dynamic system optima is built up in the frame of CTM, with the objective of minimizing the evacuation clearance time, with the main conditions of model contained flow conservation, flow transmission and sending-receiving balance in cell network.

Firstly, we analyses the crossing elimination strategy. The basic rule of carrying out this technique is to make the flow through an intersection be uninterrupted, such as blocking lane entries and limiting flow directions by the use of traffic control device, such as barrier and other police support. Take a four-leg intersection as an example, there are 16 conflict points in the signal intersection including crossing points, and all of them can be eliminated as shown in the Fig 1.

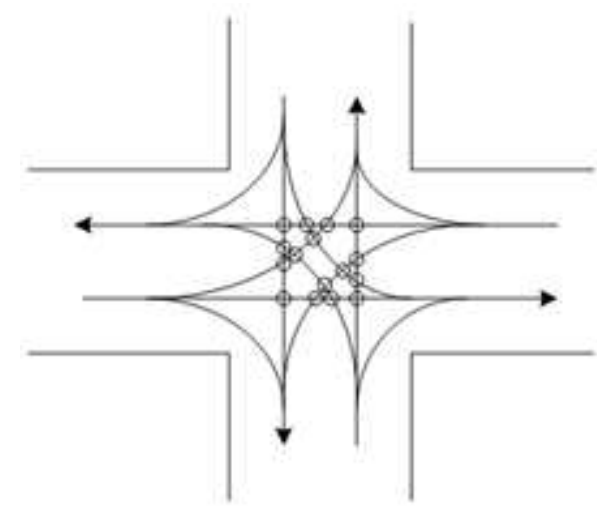

\section{Conflict points}

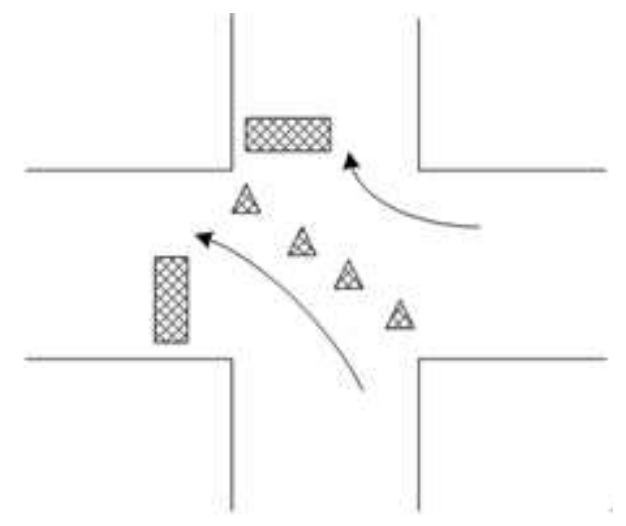

Road barriers

Fig. 1 Traffic organization strategies in a four-leg intersection

Secondly, we describe the cell network description. The way to express the cell network can be found in our past work ${ }^{[8]}$. An example of the conventional intersection is shown in Fig.2, and we can get the cell-connection graph of the road network in this way. In order to facilitate the evacuation study, the single destination is considered here.
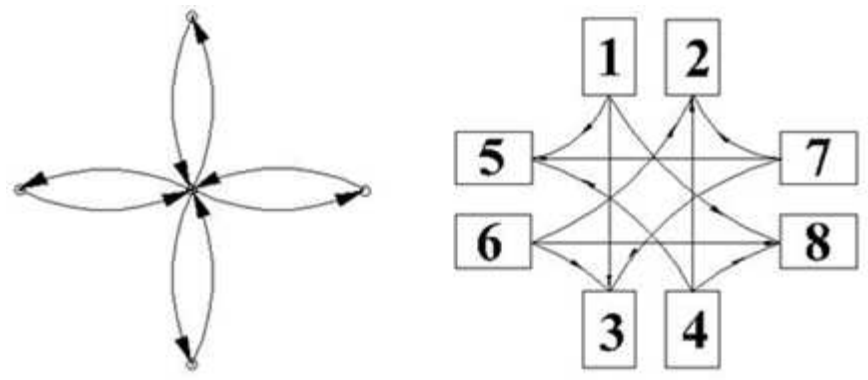

Fig.2 Cell-connection model of a four-leg intersection 
Now, we can formulate the system-optimum optimization model, which is based on dynamic traffic assignment of the road work. This modified CTM model can accurately capture the randomness and evolution process of the traffic flow in the time and space. The objective of the model is to make the total evacuation time minimum. Total travel time of the system can be explained as follows:

$$
\begin{aligned}
& \min Z=\sum_{t \in \Omega /\{0\}} \sum_{i \in \mho /\left\{\mho_{p}\right\}} x_{i}^{t} \\
& \text { s.t. } x_{i}^{t+1}=x_{i}^{t}+\sum_{k \in \psi^{-}(i)} y_{k i}^{t}-\sum_{j \in \psi(i)} y_{i j}^{t}+r_{i}^{t} \quad \forall t \in \Omega /\{T\}, \forall i \in \mho \\
& \sum_{j \in \psi(i)} y_{i j}^{t} \leq x_{i}^{t} ; \forall t \in \Omega /\{T\}, \forall i \in \mho \\
& \sum_{j \in \psi(i)} y_{i j}^{t} \leq Q_{i}^{t} ; \forall t \in \Omega /\{T\}, \forall i \in \mho \\
& \sum_{k \in \Psi^{-}(i)} y_{k i}^{t} \leq \delta_{i}^{t}\left[N_{i}^{t}-x_{i}^{t}\right] ; \forall t \in \Omega /\{T\}, \forall i \in \mho \\
& \sum_{k \in \psi^{-}(i)} y_{k i}^{t} \leq Q_{i}^{t} ; \forall t \in \Omega /\{T\}, \forall i \in \mho \\
& x_{p}^{T}=\sum_{t=0}^{T-1} \sum_{i \in \mho_{s}} r_{i}^{t}, p \in \mho_{p} \\
& \pi_{i j}+\pi_{m n} \leq 1 \quad \forall(i, j) \in \chi,(m, n) \in \Psi(i, j) . \\
& y_{i j}^{t} \leq M_{i j} . \pi_{i j} \forall i \in \mho \\
& x_{i}^{0}=0 \quad \forall i \in \mho \\
& x_{i}^{t} \geq 0, \forall t \in \Omega, \forall i \in \mho
\end{aligned}
$$

Equation (1) minimizes the evacuation time of the whole system, and it means the sum of every product of the number of vehicles in all cells except the sink cell $\mho_{p}$ and time interval unit $\tau$.Equation (2) denotes cell flow conservation and flow propagation equations can be express as sending and receiving vehicle constraint seperately in equation (3) and equation (4). The next equation (5) is balance constraint of the cell transmission model. Equation (6) and (7) are crossing elimination constraint. As for the former equation, it is used to ensure the rationality that only one direction can go through vehicles, because the sum of the two binary indicators is not greater than 1 for any flow directions $(i, j) \in \chi$. The latter equation constructs the relationship between binary indicator $\pi$ and the connector flow $y$, which can restrict the capacity of the direction in the connector. Equation (8) and (9) are balance constraint including non-negative and initial constraints.

The model can be formed as a mixed integer programming model with decision variables including $x_{i}^{t}, y_{i j}^{t}$ and $\pi_{i j}$. $\pi_{i j}$ is used to help to set the flow direction, which is a binary variable .We can get the number of vehicles in any cell, the optimum flow of the connecter between two cells and the organization of intersections in a determined time period.

Here, $L N(I)$ denotes lane set of road link $I \in A ; \mho(I)$ denotes cell sets of link $I ; Q_{i}$ denotes the maximum inflow of the cell in a unit time interval; $N_{i}$ denotes maximum vehicles of the link cell; $\Psi(i, j)$ denotes flow direction set where the direction clash with the direction $(i, j)$ in the intersection. 


\section{Numerical studies}

Finally, taking road network as an example to analyze and verify the proposed model, numerical experimental results show that evacuation efficiency can be improved significantly under optimal management strategy. There are 9 signalized intersections, and node no.7and no 9 are destinations. Traffic zone 1 covers the scope including node no. from 1 to 6 with 25,000 vehicles, and traffic zone 2 covers the scope including node no. from 4 to 9 with 15,000 vehicles. Here, the number of vehicles is got according to the population. Basic data of links are presented in the table.

Tab.1 Basic data of links in evacuation area

\begin{tabular}{c|c|c|c|c}
\hline no. & lanes one-way & $\begin{array}{c}\text { capacity one-way } \\
(\mathrm{veh} / \mathrm{h})\end{array}$ & $\begin{array}{c}\text { free speed } \\
(\mathrm{km} / \mathrm{h})\end{array}$ & $\begin{array}{c}\text { length } \\
(\mathrm{km})\end{array}$ \\
\hline 1,2 & 3 & 2200 & 60 & $1.8,1.1$ \\
\hline 6,7 & 3 & 2200 & 60 & $1.7,1.6$ \\
\hline 11,12 & 3 & 2200 & 60 & $1.1,2.1$ \\
\hline 3,8 & 3 & 2200 & 60 & $1.4,1.5$ \\
\hline 5,10 & 3 & 700 & 30 & $1.9,1.6$ \\
\hline
\end{tabular}

We assume that the evacuation demand is loading according to the classical type in MASSVAC. The time range is 30 minutes, and the unit time interval $\tau=1 \mathrm{~min}$, according to the basic information of the link, the road network on the left can be converted into the cell-connector model on the right in Fig.5. The no. 1 and 2 of cells are origin cells, no. 50 and 51 are destination cells, and the no.52 is sink destination cell.

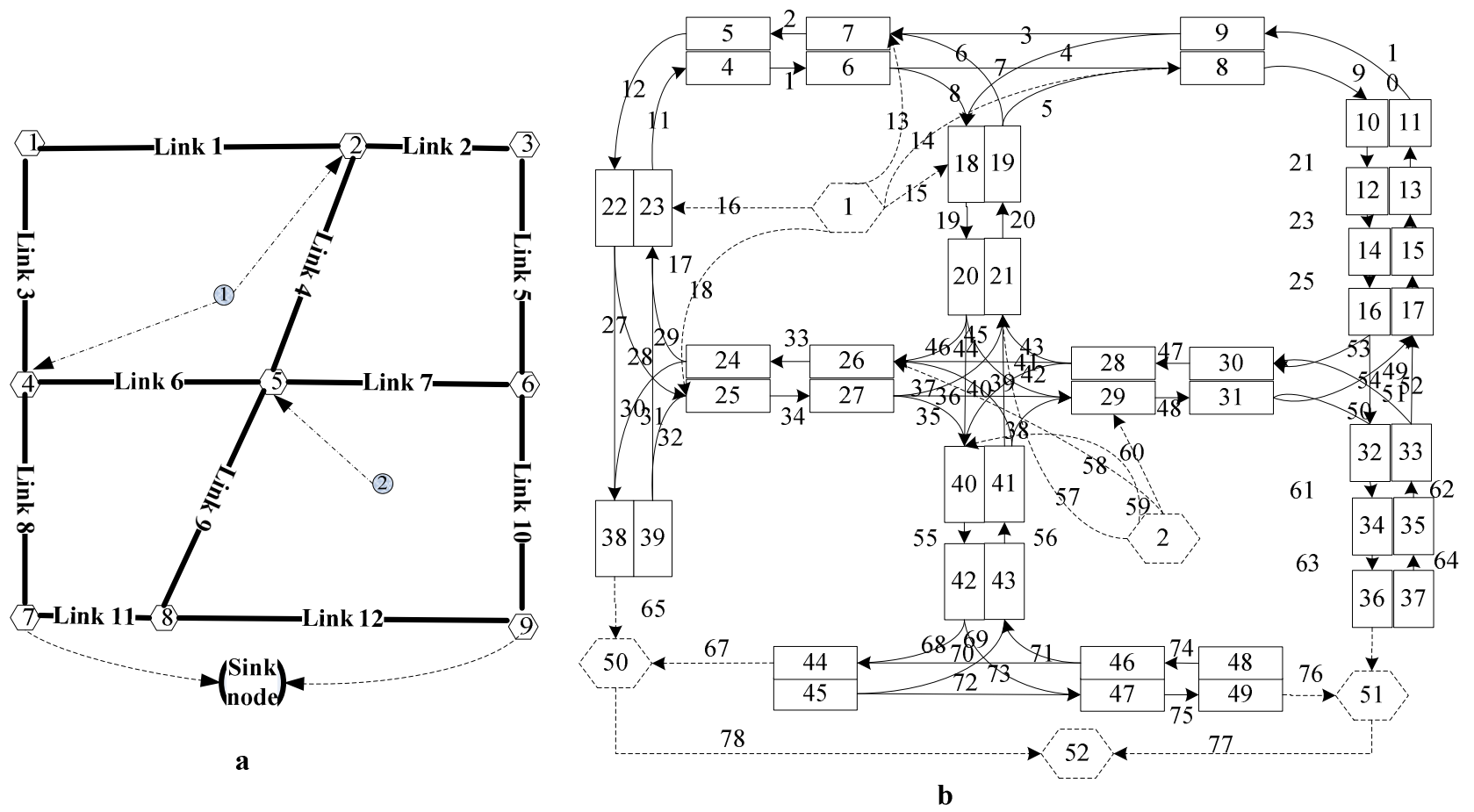

Fig.3 The cell-connector model of the evacuation road network

Based on the above data, this paper uses the Matlab and the function Miprog() to solve the model. Solution results show during 30 time intervals the, the number of total evacuation vehicles are 15738, including 10174 of destination 1 and 5564 of destination 2. Fig. 6 shows optimization layout of the evacuation area. 


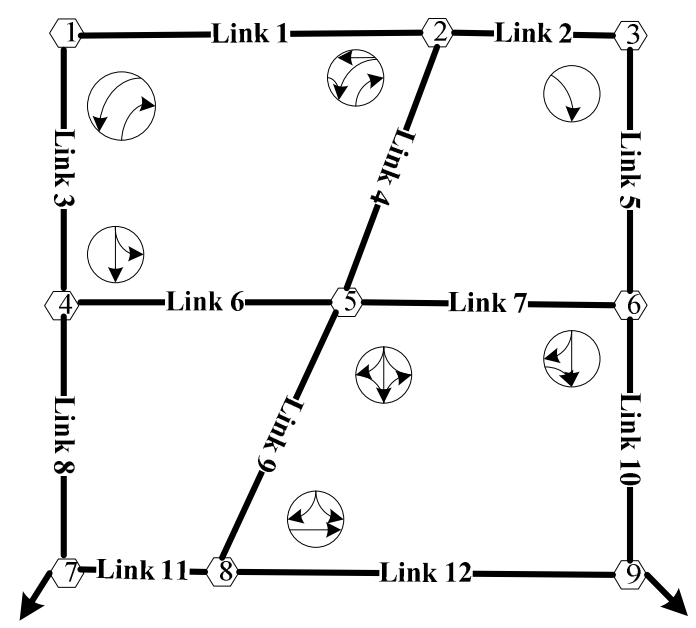

Fig.4 The setting results of crossing elimation

\section{Conclusions}

Under the conditions of emergency evacuation, applying crossing elimination is a critical and necessary measure to improve the efficiency significantly instead of the stop-and-go traffic control when drivers traverse though the intersection, because the intersection can be converted to be an uninterrupted flow facility. Our paper provides a reasonable model to get the organization and layout of the intersections, which is practical to a real evacuation plan. Urban ability dealing with all kinds of disaster may be strengthened by the implementation of scientific traffic emergency management plan. The research makes contribution to deeply understand the operation mechanism of evacuation traffic flow and provides crucial theoretical support for making evacuation traffic management strategies for decision maker. The model established in this paper is essentially a mixed integer programming model with lots of 0-1 decision variables. As the expansion of road network, the increasing in discrete time intervals, it will become more difficult to get optimization solutions. Thus, future research should be investigated on heuristic algorithm design for large-scale evacuation situation.

\section{Acknowledgements}

This research was supported by National Natural Science Foundation of China (Project No. 70973032).

\section{References}

[1] Ardekani, S.A., Hobeika, A.G.. Logistics problems in the aftermath of the 1985 Mexico City earthquake, J. Transportation Quarterly. 1998, 42 (1) 107-124.

[2] Cova, T.J., Johnson, J.P.. A network flow model for lane-based evacuation routing, J. Transportation Research Part A. 2003, 37 (7) 579-604.

[3] An Shi, Cui Jianxun, Wang Jian. An International Review of Road Emergency Transport and Evacuation $[\mathrm{J}]$. Journal of Transportation Systems Engineering and Information Technology. 2008, 8(6) 38-45.

[4] Chen Yueming, Xiao Deyun. Emergency Evacuation Model and Algorithms, J.Journal of Transportation Systems Engineering and Information Technology. 2008, 8(6) 96-100.

[5] Liu, X.M., Hu, H. Research status and prospect of emergency transportation evacuation [J]. Journal of Traffic and Transportation Engineering, 2008, 8(3) 108-121. 
[6] Liu, Y. Two-Level Integrated Optimization System for Planning of Emergency Evacuation [J]. Journal of Transportation Engineering. 2006, 132(10) 800-807.

[7] Daganzo, F C. The cell transmission model: A dynamic representation of highway traffic consistent with the hydrodynamic theory, J. Transportation research Part B. 1994, 28(4) 269-287.

[8] Cui Jianxun, An Shi, Cui Na. Dynamic regional evacuation traffic assignment based on cell transmission model. Journal of Harbin Institute of Technology. 2010, 42(1) 123-127. 